\title{
The development and psychometric properties of oral health assessment instruments used by non-dental professionals for nursing home residents: a systematic review
}

Rojina Thapa', Ritesh Chimoriya ${ }^{1,2}$ and Amit Arora ${ }^{1,3,4,5^{*}}$

\begin{abstract}
Background: Globally, oral health status of the geriatric population residing in nursing homes is poor. The integration of non-dental professionals is vital to monitor oral health, early identification and triaging of oral health problems, and timely referral to dental professionals. The aims of this systematic review were to provide a summary on the development and characteristics of oral health assessment instruments currently used by non-dental professionals for nursing home residents, and to perform a critical appraisal of their psychometric properties.
\end{abstract}

Methods: This review was conducted as per the PRISMA guidelines. CINHAL (EBSCO), Medline (Ovid), and EMBASE (Ovid) were searched systematically. Two reviewers independently screened the title, abstract, and full text of the studies as per the eligibility criteria. Studies describing oral health assessment instruments used to assess oral health of nursing home residents by non-dental professionals were included. Using a methodological framework, each instrument was evaluated for purpose, content, and psychometric properties related to validity, reliability, feasibility, generalisability, and responsiveness. Additionally, the reporting quality assessment of each included study was performed according to the SURGE guidelines.

Results: Out of the 819 screened articles, 10 studies were included in this review. The 10 identified instruments integrated 2 to 12 categories to assess oral health, which was scored on a 2 to 5 -point scale. However, the measurement content varied widely, and none were able to comprehensively measure all aspects of oral health. Three measurement approaches were identified: performance- based assessment, direct inspection of the oral health status, and interview measures. Only eight instruments provided quality assessment on the basis of validity, reliability, feasibility and generalisability, whereas three instruments- Brief Oral Health Status Examination, Dental Hygiene Registration, and Oral Health Assessment Tool reported good methodological quality on at least one assessment criteria.

(Continued on next page)

\footnotetext{
* Correspondence: a.arora@westernsydney.edu.au

'School of Health Sciences, Western Sydney University, Campbelltown

Campus, Locked Bag 1797, Penrith, NSW 2751, Australia

${ }^{3}$ Translational Health Research Institute, Western Sydney University, Locked

Bag 1797, Penrith, NSW 2751, Australia

Full list of author information is available at the end of the article
}

(C) The Author(s). 2021 Open Access This article is licensed under a Creative Commons Attribution 4.0 International License, which permits use, sharing, adaptation, distribution and reproduction in any medium or format, as long as you give appropriate credit to the original author(s) and the source, provide a link to the Creative Commons licence, and indicate if changes were made. The images or other third party material in this article are included in the article's Creative Commons licence, unless indicated otherwise in a credit line to the material. If material is not included in the article's Creative Commons licence and your intended use is not permitted by statutory regulation or exceeds the permitted use, you will need to obtain permission directly from the copyright holder. To view a copy of this licence, visit http://creativecommons.org/licenses/by/4.0/ The Creative Commons Public Domain Dedication waiver (http://creativecommons.org/publicdomain/zero/1.0/) applies to the data made available in this article, unless otherwise stated in a credit line to the data. 
(Continued from previous page)

Conclusions: None of the instruments identified in this review provided a comprehensive assessment of oral health, while three instruments appeared to be valid and reliable. Nonetheless, continuous development of instruments is essential to embrace the complete spectrum of oral health and address the psychometric gaps.

Keywords: Oral health, Geriatric assessment, Psychometrics, Reliability, Validity, Non-dental professionals

\section{Background}

There is a two-way relationship between oral health and general health [1]. Oral health refers to the condition of individual's teeth and gums, and the health of the muscles and bones present in the mouth [2]. Impaired oral health may lead to pain, discomfort, reduced chewing ability, limited food choices, poor nutritional intake, low self-esteem, social avoidance, and has a negative impact on the quality of life [3]. Moreover, poor oral health outcomes such as dental caries (tooth decay) and periodontal (gum) diseases may further increase the risk of systemic diseases [4-6].

Especially in the geriatric population, age-related degenerative changes, risk of chronic diseases, physical weakness, functional dependency, cognitive impairment, and behavioural problems act as contributing factors in the development of oral health problems [7]. Furthermore, polypharmacy, lack of dexterity, multiple systemic conditions such as diabetes, dementia, and obesity put the older adults at a high risk of oral diseases [8]. As a result, numerous cases of dental caries, xerostomia (dry mouth), oral mucosal lesions, periodontal diseases, orodental trauma, oral cancers, and frequent tooth loss are seen in older adults [9]. Several studies conducted globally suggest that about 1 in 5 older adults aged 65 and above have tooth loss [10-12], while more than half have periodontal diseases [11, 13]. This suggests that oral health problems are one of the major global health concerns with an increased prevalence of oral diseases among older people [14].

Globally, oral health status of older adults residing in nursing homes is poor [15-17]. This is because regular oral health examinations may not be available in Residential Aged Care Facilities (RACFs) and frequent commuting may be difficult due to limited mobility, cognitive impairment, and communication issues [18, 19]. It is estimated that up to $80 \%$ of nursing home residents do not receive daily oral care as they depend on the care staff as a result of their cognitive and physical limitations [20]. Studies have shown that more than $40 \%$ of the nursing home residents in Norway had unsatisfactory oral hygiene [21]; and about $70 \%$ did not receive adequate oral care in Sweden [22]. Similarly, a study conducted in Hong Kong revealed that the mean number of decayed, missing, and filled teeth (DMFT) in institutionalised older adults was 21.4 , while it was only 17.7 for those non-institutionalised [23]. This suggests that among older people, those who are residing in nursing homes are at a particularly higher risk of developing oral health problems.

Oral health assessment of all residents on a regular basis is a promising approach to delivering high quality oral health services in RACFs [24-26]. There has been a growing emphasis on providing oral health training particularly for non-dental professionals such as nursing and care staff working in RACFs [25, 27, 28]. Moreover, it is essential to ensure that oral health promotion programs are tailored to the needs of older adults and are focused on capacity building of non-dental professionals so that knowledge is effectively translated into practice $[23,29]$. The integration of front-line health care providers is also vital to monitor resident's oral health, early identification and triaging of oral health problems, and timely referral to dental professionals [30].

As most oral health assessment instruments had been developed specifically for use by dental professionals, they may be too complex for use by non-dental professionals [31]. Few instruments have been developed for use by non-dental professionals such as Oral Assessment Guide (OAG) [32] and the Holistic and Reliable Oral Assessment Tool (THROAT) [33]; however, their primary focus is on hospital and rehabilitation settings and have not been tested in residential care settings. Some of the oral health assessment instruments designed for use in RACFs include Brief Oral Health Status Examination (BOHSE) [34], Activities of Daily Oral Hygiene (ADOH) [35], Mucosal Plaque Score (MPS) [36], and Oral Health Assessment Tool (OHAT) [37]. In order to provide credible evidence to inform clinical practice and oral health policies, assessing and testing the psychometric properties of these instruments is essential. These include: validity- the extent to which an instrument measures what it is intended to measure [38], reliability- the extent to which the measurement is consistent and free from errors [39], feasibility- the administration of the instrument and the requirements associated with it [40], generalisability- the application of the instrument in different populations and settings [40], and responsivenessthe ability of an instrument to identify the important clinical changes over time within the individual [41].

In 2005, a systematic review of oral health assessment by nurses and carers for residents with dementia in 
RACFs was conducted [24]. The study indicated a shortage of validated and reliable tools available for use by non-dental professionals in RACFs. However, several oral health assessment instruments have been developed in the last two decades, and although a few of these instruments have been tested on their reliability and validity [33, 42, 43], a summary of the instruments' development and psychometric properties have not been published. Therefore, the aims of this systematic review were to provide a summary on the development and characteristics of oral health assessment instruments currently used by non-dental professionals for nursing home residents, and to perform a critical appraisal of the psychometric properties related to validity, reliability, feasibility, generalisability, and responsiveness of these instruments. Additionally, this review also assessed the reporting quality of the existing literature addressing the development and validation of these instruments.

\section{Methods}

This review was conducted in accordance with the Preferred Reporting Items for Systematic Reviews and Meta-Analysis (PRISMA) guidelines [44] (Additional file 1: Appendix 1). The protocol of this systematic review was registered with PROSPERO International Prospective Register of Systematic Reviews (2020: PROSPERO CRD42020134034) [45].

\section{Eligibility criteria \\ Inclusion criteria}

- Written in English language.

- Studies targeting the geriatric population of RACFs.

- Studies describing tools used by non-dental professionals.

- Studies focused on tools used to assess oral health.

\section{Exclusion criteria}

- Studies focused on the geriatric population in community settings.

- Studies indicating tools used solely by oral health professionals such as dentists and dental hygienists.

- Studies describing tools used solely in hospitals and rehabilitation units.

- Studies focused solely on the oral health related quality of life.

- Studies focused exclusively on the population groups with specific medical conditions.

\section{Information sources}

A large-scale search was conducted in three electronic databases- CINHAL (EBSCO), Medline (Ovid), and EMBASE (Ovid) using the specified search strategy without any restrictions on publication date (i.e. from the time of inception to present) and study type. Further, reference lists of all articles identified from the electronic databases were screened and a manual search was performed for previous systematic reviews. The initial search was conducted from 5 March 2019 and then updated on 12 August 2020. The studies were restricted to English language publications.

\section{Search strategy}

The Population Intervention/Exposure Comparator Outcome Study design (PICOS) [46] criteria were applied to design the key concepts and related additional terms. A combination of specific medical subject headings $(\mathrm{MeSH})$, terms and keywords related to oral health, the geriatric population, and RACFs were devised with the assistance from an expert Health Sciences Librarian (Additional file 2: Appendix 2). The Boolean operators 'and' and 'or' were used to narrow down and widen the search scope. The pilot search was pre-tested in the Medline (Ovid) database and was subsequently adapted to the syntax and subject headings of the other databases employed. The search strategy is provided in Additional file 3: Appendix 3.

\section{Study selection}

All studies retrieved from the electronic databases were exported to a reference manager software Endnote X9 for elimination of duplicates, screening, and selection. Two reviewers (RT and AA), in accordance with the eligibility criteria, independently screened the searches and filtered the manuscripts by title and abstract relevance. Studies that intended to measure the oral health status of the geriatric population residing in RACFs or to develop a new oral health assessment tool used by non-dental personnel were read in full text. Any disagreements were resolved through discussion with a third reviewer (RC). The studies that were read in full text and found not to meet the inclusion criteria have their reason/s for exclusion reported in Additional file 4: Appendix 4.

\section{Data extraction process}

A standardised data extraction form was developed to evaluate all oral health assessment instruments, using a methodological framework $[47,48]$ established for the evaluation of health assessment indices as a reference. Necessary adaptations were made to the categories within the framework so that the appraisal was relevant for oral health assessment instruments. The data extraction form was pilot tested on two studies to ensure it met the review objectives and all relevant information were recorded consistently. Data from all included studies were extracted independently by two reviewers (RT and AA). Information on country of origin, publication 
year, authors, type of tool, purposes, developers, method of development, administration procedure, estimated duration for assessment, and scoring categories for each assessment tool were extracted. Furthermore, studies were assessed for psychometric analysis, and information related to validity, reliability, feasibility, generalisability, and responsiveness were extracted.

\section{Assessment of reporting quality}

The reporting quality assessment of each included study was performed according to the specifications of the Reporting Guidelines for Survey Research (SURGE) [49]. The SURGE is an adequate and appropriate tool to appraise the reporting quality in surveys and to gain detailed information on the characteristics of the survey instruments used [50]. The studies were appraised in terms of eight categories: background, methods, sample selection, characteristics of the research tool, results, response rates, interpretation and discussion, and ethics and disclosure [49]. The degree to which the intention of each study matched were then reported as partial, inadequate, and adequate. Two reviewers (RT and AA) independently assessed the methodological quality.

\section{Data synthesis}

Following data extraction, a narrative was created to provide a descriptive synthesis of the included studies in two steps. The first task was to assess the purpose and content of all identified instruments including sampling frame, settings, and oral health domains targeted in each study. The second task was to ascertain the psychometric properties- reliability, validity, feasibility, generalisability, and responsiveness of each instrument.

\section{Validity}

The determination of validity for a particular instrument depends upon a variety of contexts and the purpose of the research [40]. It is important to define content, face, and construct validity for a newly developed instrument [39]. Content validity is associated with the process of planning and developing an instrument and looks at the extent to which the content of the instrument reflects the concept that is being measured [40]. Face validity is where an instrument appears to test what it is intended to measure. Construct validity assesses the degree to which an instrument measures what it is supposed to measure and can be assessed through confirmatory factor analysis, hypothesis testing, and comparing and examining instrument associations with existing instruments [40].

\section{Reliability}

Various ways of estimating reliability include assessing internal consistency of each item in the instrument (internal consistency reliability), consistency of measurement over time (test-retest reliability), establishing degree of agreement between either two or more examiners (inter-rater reliability), and assessing result consistency measured by the same examiner (intra-rater reliability) [39].

\section{Feasibility}

Feasibility can be judged based on parameters such as estimated time, resources required, and to what extent the instruments are suitable to the users and recipients [51].

\section{Results}

\section{Results of the search}

A total of 819 articles were retrieved from the electronic databases and manual search. Of these, 374 articles were eliminated due to duplication. After reviewing the abstracts, 413 articles were excluded as they did not measure oral health and only reported on the development and validation of oral health instruments used in RACFs. Further 4 studies were removed due to publication only in non-English language, and 2 studies were removed due to accessibility issues, despite repeated attempts to contact the authors. A total of 26 full-text studies were assessed by two reviewers (RT and AA), which resulted in further exclusion of 16 studies based on the eligibility criteria. The reasons for exclusion are presented in Appendix 4. Finally, a total of 10 studies were included in this systematic review. The results of the search process are outlined in the PRISMA flow diagram (Fig. 1).

\section{Characteristics of oral health assessment instruments}

Table 1 illustrates the overview of oral health assessment instruments included in this review. The 10 identified instruments were developed between 1990 and 2017. All included studies originated from high income countries such as Sweden [56], Norway [36, 52], United States [34, 35, 53, 55], Australia [43], and Japan [31, 54]. All instruments integrated 2 to 12 categories to assess oral health, which was scored on a 2 to 5 -point scale.

The characteristics of oral health assessment instruments are outlined in Table 2. The primary purpose of all included studies was to develop an instrument for nurses to assess oral health of aged care residents. Additionally, six studies [34, 36, 43, 52-54] stated testing and validation of instruments by nurses and care workers in RACFs as their primary objective. Most of the instruments were developed by a panel of experts in geriatrics, dentistry, nursing, and in consultation with the users. Four instruments were developed by modifying the existing instruments- OHAT from BOHSE [43]; Dental Hygiene Registration (DHR) from Simplified Oral Hygiene Index (OHI-S), MPS, and Revised Oral Assessment Guide (ROAG) [52]; Revised Oral Assessment 


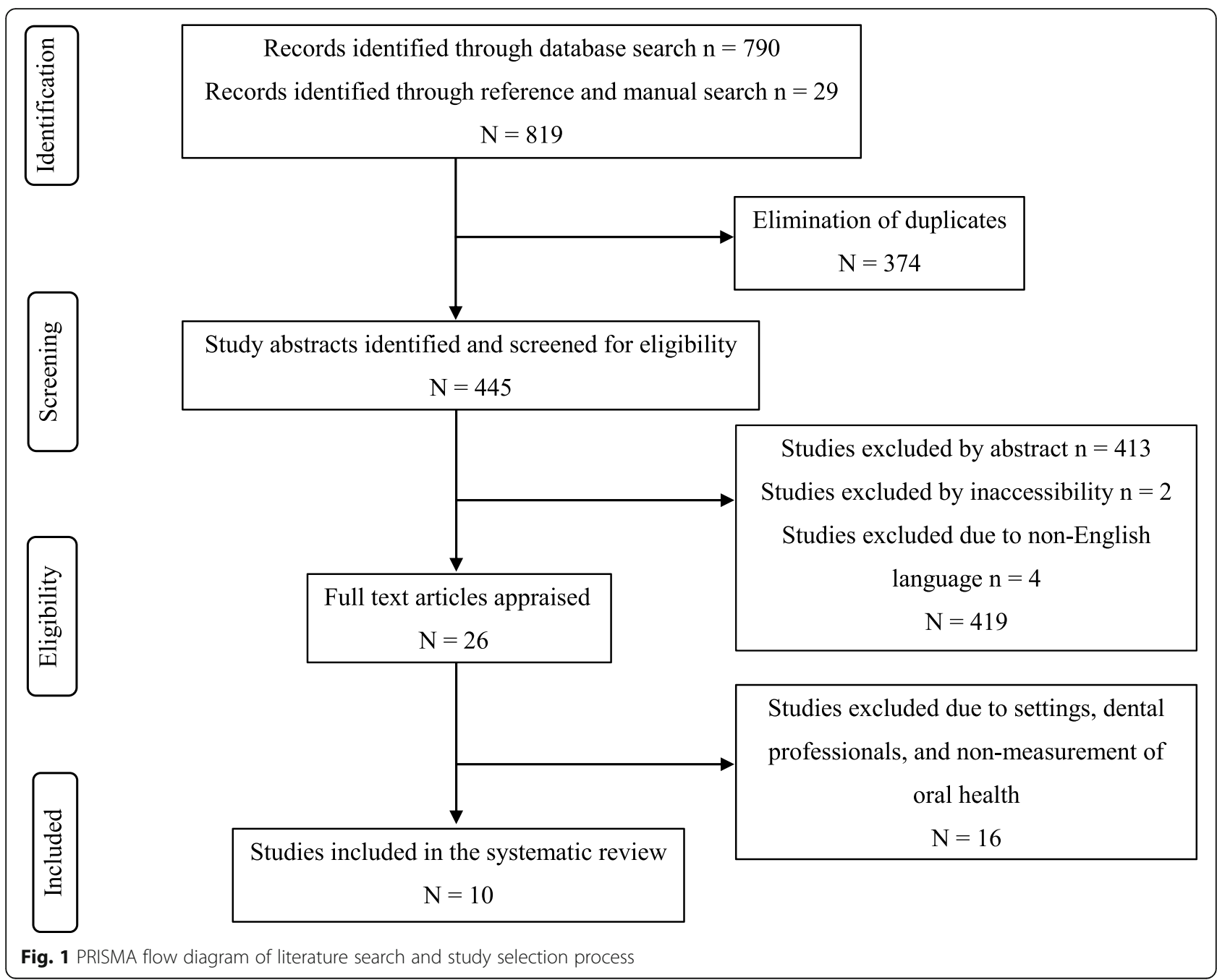

Table 1 Overview of oral health assessment instruments

\begin{tabular}{|c|c|c|c|c|}
\hline Instrument & Year & Country of origin & Authors & Type of instrument \\
\hline $\mathrm{ADOH}[35]$ & 2001 & United States & Bauer et al. & 4 categories scored on 5-point scale \\
\hline BOHSE [34] & 1995 & United States & Kayser-Jones et al. & 10 categories scored on 3-point scale \\
\hline DHR [52] & 2016 & Norway & Fjeld et al. & 2 categories scored on 3-point scale \\
\hline GOHAI [53] & 1990 & United States & Atchison et al. & 12 categories scored on 3-point scale \\
\hline MPS [36] & 1999 & Norway & Henriksen et al. & 2 categories scored on 4-point scale \\
\hline OAS [54] & 2017 & Japan & Shizuko et al. & 9 categories scored on 3-point scale \\
\hline OHAT [43] & 2004 & Australia & Chalmers et al. & 8 categories scored on 3-point scale \\
\hline OHSTNP [31] & 2017 & Japan & Tsukada et al. & 12 categories scored on 3-point scale \\
\hline RAI-MDS [55] & 1990 & United States & Morris et al. & 2 sections with yes/no questions \\
\hline ROAG-J [56] & 2016 & Sweden & Johansson et al. & 8 categories scored on 4-point scale \\
\hline
\end{tabular}

ADOH: Activities of Daily Oral Hygiene, BOHSE: Brief Oral Health Status Examination, DHR: Dental Hygiene Registration, GOHAl: Geriatric Oral Health Assessment Index, MPS: Mucosal Plaque Score, OAS: Oral Assessment Sheet, OHAT: Oral Health Assessment Tool, OHSTNP: Oral Health Screening Tool for Nursing Personnel, RAI-MDS: Resident Assessment Instrument-Minimum Data Set, ROAG-J: Revised Oral Assessment Guide-Jonkoping 
Guide-Jonkoping (ROAG-J) from ROAG and OAG [56]; and Oral Health Screening Tool for Nursing Personnel (OHSTNP) from OHAT and Oral Screening Sheet [31]. In terms of administration, most instruments used inspection and palpation for examination of oral health status and scored using a point scale. Geriatric Oral Health Assessment Index (GOHAI) [53] used questionnaire-based oral health assessment, while $\mathrm{ADOH}$ [35] used taskperformance oriented questions to assess oral health.

The following section summarises the development and characteristics of each oral health assessment instrument included in this review.

\section{Activities of Daily Oral Hygiene (ADOH)}

$\mathrm{ADOH}$ was developed to determine and monitor the functional dependency of an individual to operate the aids used in oral self-care [35]. The four domains for assessing the dependency function of oral self-care activities include flossing, tooth brushing, topical fluoride application, and oral rinses. A score ranging from 0 to 4 is allocated for each task, which takes about 5 to $15 \mathrm{~min}$ to complete. For edentulous older adults, brushing and oral rinsing tasks are rated with the total dependency score of 8 . On the basis of the total score, an individual is classified as independent, partly dependent, or fully dependent.

\section{Brief Oral Health Status Examination (BOHSE)}

BOHSE was developed to measure the oral health conditions of cognitively impaired and unimpaired residents of aged care facilities by the care providers [34]. The ten assessment items related to oral health and function include lymph nodes, lips, tongue, tissue inside cheek, floor and roof of the mouth, gums between teeth and/or under the dentures, saliva, condition of natural teeth and dentures, pairs of teeth in chewing position, and oral cleanliness. Each item consists of three descriptors and is scored from 0 to 2 . The summative score ranges from 0 (very healthy) to 20 (very unhealthy).

\section{Dental Hygiene Registration (DHR)}

DHR is a dental hygiene assessment scale designed to describe the individual's dental hygiene and to evaluate the nurse's own performance in delivering dental hygiene [52]. DHR was developed considering the benefits and strengths of OHI-S, MPS, and ROAG. The presence of visible dental plaque on all tooth surfaces is examined and scored to assess oral hygiene. The upper and lower jaw are scored separately, and then summed to get an overall score of $0-4$. If one of the jaws is edentulous, the other jaw is scored and multiplied by two.

\section{Geriatric Oral Health Assessment Index (GOHAl)}

GOHAI is a questionnaire-based tool designed to assess the oral health of older adults [53]. It consists of twelve items related to physical function, psychological function, and pain or discomfort; and includes both positive and negative items rated on a 3-point Likert scale as 1always, often; 2- sometimes, seldom; and 3- never. It is recommended as a quick and easy instrument for use by nurses in RACFs as it helps to collect oral complaints and decide appropriate dentist referral [57]. GOHAI has been translated into different languages- Chinese [58], Dutch [59], French [60], Swedish [61], and Turkish [57] since its development.

\section{Mucosal Plaque Score (MPS)}

MPS measures only two parameters- degree of inflammation of the mucosa and the amount of plaque deposited around the teeth or on dentures, which facilitates a quick overall evaluation of the oral health condition and oral hygiene [36]. The mucosal and plaque score are evaluated separately on the basis of four criteria- normal appearance, mild inflammation, moderate inflammation, and severe inflammation; and no visible plaque, small amounts of hardly visible plaque, moderate amounts of plaque, and abundant amounts of plaque, respectively. The scores range from 1 to 4 for each parameter which gives a total MPS score ranging from 2 to 8 .

\section{Oral Assessment Sheet (OAS)}

OAS measures the oral hygiene in terms of tooth and gingival cleanliness, coating of tongue, and bad breath; whereas oral function is measured in terms of jaw opening, tongue thrust, dry mouth, and chewing and biting function of teeth and dentures [54]. The examiner rates the oral hygiene, biting and chewing, and oral function each with three items using a grading scale: A- poor oral condition which needs immediate improvement; B- optimal oral condition yet some improvement is required; and $\mathrm{C}$ - oral condition with minimal problems. A numerical score 2, 1 and 0 is assigned to grade A, B and C respectively.

\section{Oral Health Assessment Tool (OHAT)}

OHAT is a modified version of BOHSE and evaluates the oral health status of residents of RACFs including those with cognitive impairment [43]. It was designed to measure the oral health status, monitor oral hygiene, and identify the referral need based on the eight specified categories- lips, tongue, gums and tissues, saliva, natural teeth, dentures, oral cleanliness, and dental pain. Each category is rated on a 3-point scale: 0- healthy, 1oral changes, and 2- unhealthy. OHAT is an easy to use tool for non-dental personnel ranging from carers to nurses. 
Table $\mathbf{2}$ Characteristics of oral health assessment instruments

\begin{tabular}{|c|c|c|c|c|c|}
\hline Tools & Purpose & $\begin{array}{l}\text { Expertise of } \\
\text { developers }\end{array}$ & Development & Administration & Scoring \\
\hline $\begin{array}{l}\text { ADOH } \\
{[35]}\end{array}$ & $\begin{array}{l}\text { To assess the physical } \\
\text { ability to manipulate the } \\
\text { aids used in oral self-care } \\
\text { and to measure the return } \\
\text { to function in response to } \\
\text { care intervention and re- } \\
\text { habilitative services. }\end{array}$ & $\begin{array}{l}\text { Panel of expertise from } \\
\text { geriatric dentistry. }\end{array}$ & $\begin{array}{l}\text { Conceptualised from the } \\
\text { classification scheme used } \\
\text { in medicine i.e. Activities } \\
\text { of Daily Living. }\end{array}$ & $\begin{array}{l}\text { The sequence of } \\
\text { interviewing instructions is } \\
\text { given in the assessment } \\
\text { instrument to guide the } \\
\text { examiner in monitoring } \\
\text { the individual's abilities in } \\
\text { performing each task. }\end{array}$ & $\begin{array}{l}4 \text { categories scored on 5- } \\
\text { point scale from 0-4. } \\
0 \text { : for performing each } \\
\text { step without any help; } \\
\text { 1: requires a device to } \\
\text { enhance the performance; } \\
\text { 2: expends } 50 \% \text { or more } \\
\text { effort in task completion; } \\
\text { 3: expends less than 50\% } \\
\text { effort in task completion; } \\
\text { 4: for total assistance in } \\
\text { performing task. } \\
\text { Total score of 16. Classifies } \\
\text { an individual as } \\
\text { independent, partly } \\
\text { dependent and } \\
\text { dependent. }\end{array}$ \\
\hline
\end{tabular}

BOHSE To evaluate the oral health Registered nurses,

[34] status of both cognitively impaired and unimpaired residents by nursing staff. dentists, certified nursing assistants, and licensed vocational nurses. American Dental
Developed through a review of available oral consultation with dental faculty, and recommendations from Association. assessment guides,

Examination is carried out in the same order as given in instrument guide starting from inspection and palpation of lymph nodes to observing oral cleanliness. Tongue blades, light, disposable gloves, and gauze squares were used as per need.

Literature review on existing instruments. Benefits and strengths from OHI-S, MPS, and ROAG were considered and DHR criteria were discussed with a panel of expertise.

Assessment is conducted using a pen torch light source. Entire tooth surfaces of upper jaw are examined first and then the lower jaw for the presence of plaque (yes/ no).
10 categories scored on 3point scale from 0 to 2 .

0 : indicating healthy end;

2: unhealthy end of the scale.

Total score of 20 which ranges from 0 : very healthy to 20: very

unhealthy.

A higher cumulative score reflects the presence of many oral health problems.

2 categories scored on 3 point scale from 0 to 2 . Total score of 4 , where 0 : teeth without plaque represent optimal; 1 : visible plaque on one or more teeth represent increased risk; 2-4: visible plaque on more than half of the teeth represent risk of oral health diseases. Lower and upper jaw are scored separately.

12 categories scored on 3point scale from 1 to 3 .

1: always, often; 2 : sometimes, seldom; 3: never. High score represents good oral health and low score represents oral health problems.

Examination is performed in normal daylight with the aid of an artificial light source. Intraoral examination is performed with the help of dental mirrors.

2 categories scored on 4-

point scale from 1 to 4 . $\begin{array}{ll}\text { and oral hygiene in } & \text { gerontology and } \\ \text { hospitals and other } & \text { dentistry faculty. }\end{array}$ institutions.

OAS [54] To enhance the oral Expertise comprising of Oral assessment items health of older adults who dentists, dental need nursing care. performed without using any special instrument.
Oral examination is
4. acceptable; $5-6$ :

unacceptable; 7-8: poor. Mucosal and Plaque Score are calculated separately.

9 categories scored on 3point scale from A-C A: poor oral condition which needs immediate were decided by the focus group of expertise after the literature review hygienists, medic social workers, and 
Table 2 Characteristics of oral health assessment instruments (Continued)

\begin{tabular}{|c|c|c|c|c|c|}
\hline Tools & Purpose & $\begin{array}{l}\text { Expertise of } \\
\text { developers }\end{array}$ & Development & Administration & Scoring \\
\hline & & medical doctors. & $\begin{array}{l}\text { on assessment of oral } \\
\text { health. }\end{array}$ & & $\begin{array}{l}\text { improvement; B: an } \\
\text { optimal level, yet } \\
\text { improvement is needed; } \\
\text { C: condition with } \\
\text { minimum problems. } \\
\text { Grade A, B and C } \\
\text { represent Score } 2,1 \text { and } 0 . \\
\text { High score indicates poor } \\
\text { oral health, which requires } \\
\text { professional interventions. }\end{array}$ \\
\hline $\begin{array}{l}\text { OHAT } \\
{[43]}\end{array}$ & $\begin{array}{l}\text { To assess the oral health } \\
\text { status by carers in } \\
\text { residential care facilities. }\end{array}$ & $\begin{array}{l}\text { Group of expertise } \\
\text { from geriatric dentistry, } \\
\text { dementia care, nurses, } \\
\text { dentists, dental } \\
\text { hygienists, carers, and } \\
\text { RACFs. }\end{array}$ & $\begin{array}{l}\text { Developed by modifying } \\
\text { BOHSE after review of } \\
\text { literature on oral } \\
\text { assessment tools and } \\
\text { suggestions from the } \\
\text { group of expertise from } \\
\text { Australia and United } \\
\text { States. }\end{array}$ & Not stated. & $\begin{array}{l}8 \text { categories scored on } 3- \\
\text { point scale from } 0 \text { to } 2 \text {. } \\
0 \text { : healthy; } 1 \text { : oral changes; } \\
\text { and } 2 \text { : unhealthy. } \\
\text { Total score is calculated by } \\
\text { summing scores from } \\
\text { each category. }\end{array}$ \\
\hline $\begin{array}{l}\text { OHSTNP } \\
{[31]}\end{array}$ & $\begin{array}{l}\text { To assist nursing staff of } \\
\text { long-term care in identify- } \\
\text { ing dental referral needs. }\end{array}$ & $\begin{array}{l}\text { Group of nurses, } \\
\text { dentists, and } \\
\text { caregivers. }\end{array}$ & $\begin{array}{l}\text { Developed by combining } \\
\text { and modifying OHAT and } \\
\text { Oral Screening Sheet. }\end{array}$ & $\begin{array}{l}\text { General inspection by } \\
\text { using a penlight, tongue } \\
\text { depressor, and dental } \\
\text { mirrors. }\end{array}$ & $\begin{array}{l}12 \text { categories scored on } 3- \\
\text { point scale from } 0 \text { to } 2 . \\
0 \text { : good; } 1 \text { : fair; and } 2 \text { : } \\
\text { poor. } \\
\text { Questions related to the } \\
\text { need for referral and } \\
\text { reason of assessment are } \\
\text { added at the bottom of } \\
\text { the screening tool. }\end{array}$ \\
\hline $\begin{array}{l}\text { RAI-MDS } \\
\text { [55] }\end{array}$ & $\begin{array}{l}\text { To collect minimum } \\
\text { amount of data regarding } \\
\text { resident's strengths, needs, } \\
\text { and potential risk to plan } \\
\text { and monitor individualised } \\
\text { care in long term care } \\
\text { setting. }\end{array}$ & $\begin{array}{l}\text { Clinicians and } \\
\text { researchers from } \\
\text { nursing, social work, } \\
\text { medicine, physical, } \\
\text { occupational and } \\
\text { speech therapy, and } \\
\text { nutrition disciplines. }\end{array}$ & $\begin{array}{l}\text { Developed by extensive } \\
\text { review and revision of the } \\
\text { assessment instrument } \\
\text { and developing multiple } \\
\text { drafts of MDS consulting } \\
\text { with experts and a basic } \\
\text { testing of the instrument. }\end{array}$ & $\begin{array}{l}\text { Nurses complete the } \\
\text { assessment form through } \\
\text { resident's records, direct } \\
\text { observation, and } \\
\text { conversation with } \\
\text { residents. }\end{array}$ & $\begin{array}{l}2 \text { sections pertaining to } \\
\text { oral health consisting of } \\
\text { yes/no items. } \\
\text { All responses indicating } \\
\text { potential oral problems } \\
\text { requires automatic dental } \\
\text { referral. }\end{array}$ \\
\hline $\begin{array}{l}\text { ROAG-J } \\
{[56]}\end{array}$ & $\begin{array}{l}\text { To assess the oral health } \\
\text { status of elderly people in } \\
\text { a daily nursing care. }\end{array}$ & Not stated. & $\begin{array}{l}\text { Developed by revising } \\
\text { ROAG, which in turn is } \\
\text { revised from OAG. }\end{array}$ & $\begin{array}{l}\text { Examination is performed } \\
\text { following the manual } \\
\text { provided in ROAG-J. }\end{array}$ & $\begin{array}{l}8 \text { categories scored on } 4- \\
\text { point scale from } 0 \text { to } 3 . \\
0 \text { and } 1 \text { : no intervention; } \\
2 \text { : preventive care action } \\
\text { by nurses; } 3 \text { : requires } \\
\text { dentist for treatment. }\end{array}$ \\
\hline
\end{tabular}

ADOH: Activities of Daily Oral Hygiene, BOHSE: Brief Oral Health Status Examination, DHR: Dental Hygiene Registration, GOHAl: Geriatric Oral Health Assessment Index, MPS: Mucosal Plaque Score, OAS: Oral Assessment Sheet, OHAT: Oral Health Assessment Tool, OHSTNP: Oral Health Screening Tool for Nursing Personnel, RAI-MDS: Resident Assessment Instrument-Minimum Data Set, ROAG-J: Revised Oral Assessment Guide-Jonkoping

Oral Health Screening Tool for Nursing Personnel (OHSTNP) OHSTNP was developed to identify the dental referral needs of residents in a long-term care facility [31]. It measures the oral health status and oral function and consists of twelve categories (A-L): A to $\mathrm{G}$ are related to oral health and are modified from OHAT; $\mathrm{H}$ to $\mathrm{L}$ are related to oral function and are modified from Oral Screening Sheet; and $\mathrm{K}$ and $\mathrm{L}$ indicate the response from residents or staff. Each category consists of three descriptors and are scored on a 3 -point scale: 0 (good), 1 (fair), and 2 (poor).

Resident Assessment Instrument-Minimum Data Set (RAIMDS)

MDS is an assessment instrument consisting of minimum number of items; whereas, RAI refers to the assessment system and guidelines of care planning [62]. RAI-MDS was revised to RAI-MDS version 2.0 by retaining the previously tested instrument while modifying and adding new items. It consists of seventeen sections (A-Q) with 400 data items related to health and care at an individual resident level. Among them, two sections of MDS are related to oral health section: L (oral/nutritional) and $\mathrm{M}$ (oral/dental). Oral/nutritional items include oral problems, height and weight, nutritional problems, and nutritional approaches; and oral/ dental status include oral conditions in terms of debris, denture, natural teeth, inflammation of gums, cleanliness, and dental caries. The oral health items were not changed for MDS v2.0, except that they are now included in section $\mathrm{K}$ and $\mathrm{L}$ [63]. The responses that suggest oral problems or risk are marked as ' $\bullet 15$ ', which 
indicates dental referral according to the resident assessment protocol and a system of follow-up instructions [63]. It is currently used in Canada, United States, England, Germany, Japan, Australia, New Zealand, Sweden, Norway, France, Spain, and Belgium $[64,65]$.

\section{Revised Oral Assessment Guide - Jonkoping (ROAG-J)}

ROAG-J was developed to measure the oral health status of nursing home residents in daily nursing care. It is an updated version of ROAG [42], which is in turn revised from OAG [32]. ROAG-J describes the oral health by assessing the state of lips, voice, mucous membranes, gums, teeth, dentures, saliva, and tongue. It is rated on a $0-3$ grade scale, where Grade 0 and 1 means no intervention is required, Grade 2 means an intervention is required by the nursing staff, and Grade 3 means an intervention is required by a dentist. The scores from all categories are summed up to get the potential score ranging from 0 to 27 and a higher score implies poor oral health [56].

\section{Psychometric properties of oral health assessment instruments}

Table 3 summarises the psychometric analysis of oral health assessment tools. Out of the ten identified instruments, eight instruments- BOHSE [34], DHR [52], GOHAI [53], MPS [36], OAS [54], OHAT [43], OHSTNP [31], and RAI-MDS [55] provided quality assessment on the basis of validity, reliability, feasibility and generalisability. Overall, three instruments- BOHSE [34], DHR [52], and OHAT [43] reported good methodological quality on at least one assessment criteria and appeared to be valid and reliable assessment tools for use by non-dental professionals to assess the oral health of nursing home residents. The following section summarises the psychometric properties of each oral health assessment instrument included in this review.

\section{Validity}

Six instruments- ADOH [35], ROAG-J [56] OAS [54], RAI-MDS [55], OHSTNP [31] and MPS [36] did not establish validity of the instrument which showed inadequate quality in methodology. Only three instrumentsBOHSE [34], GOHAI [53], and OHAT [43] reported on content validity; two instruments- BOHSE [34] and OHAT [43] reported on face validity; and one instrument- DHR [52] established construct validity.

DHR showed a good correlation with reported gold standards MPS [36] and Debris Index from OHI-S [52]. GOHAI showed a significant association with selfreported dental health; however, it showed a weaker correlation with clinical measures except for the number of teeth [53]. All items in OHAT [43] were not assessed and it did not reflect the construct to be measured comprehensively. In RAI-MDS 2.0, oral/dental items lacked validity as it under detects the oral/dental problems as compared to the clinical assessment by dental professionals [66].

\section{Reliability}

Two instruments- ADOH [35] and ROAG-J [56] did not report on reliability. Two instruments- OHAT [43] and BOHSE [34] assessed stability of the instrument by testretest reliability. OHAT [43] failed to show correlations over time, assessments were repeated at 3 and 6 months, hence the methodological quality was poor. Whereas BOHSE [34] reported moderate sample size and unweighted kappas with high test-retest reliability. The percent agreement for the individual items of BOHSE varied from 50.5 to 98.0 , and unweighted kappas ranged from 0.09 to 0.82 , which showed a statistical significance [34]. Assessment of measurement properties on individual items of OHAT ranged from intra-rater reliability of $74.4 \%$ for oral cleanliness to $96.6 \%$ for a referral to the dentist; and unweighted kappas ranged from 0.51 to 0.80 indicating substantial agreement, whereas percent agreement between nurses ranged from $72.6 \%$ for oral cleanliness to $92.6 \%$ for dental referral and unweighted kappas varied from 0.48 to 0.80 showing substantial inter-rater agreement.

For DHR [52], inter-rater and intra-rater reliability were scored, where unweighted kappas was 0.7 for the dental hygienist and 0.8 for clinical nurse, which showed significant agreement between the examiners. However, oral/dental items in RAI-MDS [67] showed poor interrater agreement. The percent agreements between the examiners in OHSTNP were statistically significant for the categories E-L but the kappa values (0.05-0.20) and observed agreement (24.6-39.1\%) for categories A-D were not significant [31]. Furthermore, two instrumentsOAS $(\alpha=0.72)$ [54], and GOHAI $(\alpha=0.79)$ [53] indicated excellent internal consistency, which was assessed by calculating Cronbach's alpha.

\section{Feasibility}

All instruments assessed the oral health status of the residents and required an examiner to administer all or some of the items in the assessment tools. Some tools required in-service education and training to perform the test, specifically for BOHSE [34], OHAT [43], OHSTNP [31], and OAS [54]. The estimated time required to complete the assessments ranged from a minimum of $1 \mathrm{~min}$ for DHR [52] to a maximum of $30 \mathrm{~min}$ for GOHAI [53].

\section{Generalisability and responsiveness}

GOHAI can be used by both dental professionals and non-dental personnel for the assessment of older adults 
Table 3 Psychometric analysis of oral health assessment instruments

\begin{tabular}{|c|c|c|c|c|c|c|c|c|c|}
\hline $\begin{array}{l}\text { Name of the } \\
\text { instrument }\end{array}$ & $\begin{array}{l}\mathrm{ADOH} \\
{[35]}\end{array}$ & $\begin{array}{l}\text { BOHSE } \\
{[34]}\end{array}$ & DHR [52] & GOHAI [53] & MPS [36] & OAS [54] & OHAT [43] & $\begin{array}{l}\text { OHSTNP } \\
\text { [31] }\end{array}$ & $\begin{array}{l}\text { RAI- } \\
\text { MDS } \\
{[55]}\end{array}$ \\
\hline
\end{tabular}

\section{Content validity}

a. Were the
methods of
selecting items
appropriate?
b. Is the
definition of
what is being
measured clearly
specified?
c. Were
intended
categories (i.e.
relevant areas to
be included and
excluded) clearly
stated?

Not Yes

stated

No Yes

No

Not stated

Not stated

Not

stated

Not stated Yes

Not stated

Not stated

Not

(n)

Not Partially Partially

stated

Partially

Not stated

Not

stated

Partially

Not

stated

No

d. Are all
relevant
components of
each category

Not stated

stated

Yes

Not stated

Not stated

Not

stated

Not stated Yes

Not stated

Not

stated

Not

stated

included?

\section{Face Validity}

a. On the face of it does it describe the

intended

purpose?

\section{Construct Validity}

$\begin{array}{lll}\begin{array}{l}\text { a. Does the } \\ \text { instrument }\end{array} & \text { Not } & \text { Not stated } \\ \text { perform in } & & \\ \text { expected ways } & & \\ \text { when compared } & & \\ \text { with other oral } & & \\ \text { health } & & \\ \text { assessment } & & \\ \text { indices? } & & \end{array}$

correlation with OHI-S

Spearman's

correlation co-

efficient Rs =

0.78 , and MPS

$\mathrm{Rs}=0.83$.

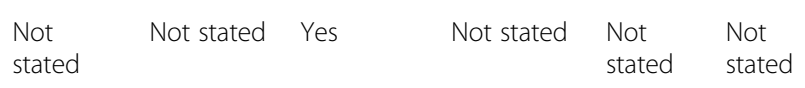

Not

stated

correlation with

Single-item self-

rating of dental

health Pearson's correlation coef-

ficients $r=0.47$

indices?
4. Reliability

a. Has the reliability been measured?

No

Yes, Test-
retest coef-
ficients
$0.79-0.88$

b. Inter-rater

No

$<0.80$

1st time kappa No

$(k)=0.5$

2nd time $\mathrm{k}=$

0.8

c. Intra-rater

reliability

d. Internal consistency

No No

No No

1 st
0.7
2nd

0.8

No
Yes

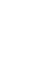

No

Yes, Cronbach's No alpha $a=0.79$
Test 1

Weighted

kappa (k)

$=0.70$;

Test $2 \mathrm{k}=$

0.77

Weighted Not stated

kappa $\mathrm{k}=$

0.62

o

Yes, No

Cronbach's

alpha $a=$

0.72

\section{Feasibility}


Table 3 Psychometric analysis of oral health assessment instruments (Continued)

\begin{tabular}{|c|c|c|c|c|c|c|c|c|c|c|}
\hline $\begin{array}{l}\text { Name of the } \\
\text { instrument }\end{array}$ & $\begin{array}{l}\text { ADOH } \\
{[35]}\end{array}$ & $\begin{array}{l}\text { BOHSE } \\
{[34]}\end{array}$ & DHR [52] & GOHAI [53] & MPS [36] & OAS [54] & OHAT [43] & $\begin{array}{l}\text { OHSTNP } \\
\text { [31] }\end{array}$ & $\begin{array}{l}\text { RAI- } \\
\text { MDS } \\
\text { [55] }\end{array}$ & $\begin{array}{l}\text { ROAG- } \\
J[56]\end{array}$ \\
\hline $\begin{array}{l}\text { a. Are special } \\
\text { skills tools and/or } \\
\text { training required? }\end{array}$ & No & $\begin{array}{l}\text { In-service } \\
\text { education }\end{array}$ & No & No & No & Yes & Yes & Yes & No & No \\
\hline $\begin{array}{l}\text { b. Is it easy to } \\
\text { perform and } \\
\text { administer? }\end{array}$ & Yes & Yes & Yes & Yes & Yes & Yes & Yes & Yes & Yes & Yes \\
\hline $\begin{array}{l}\text { c. How long } \\
\text { does it take to } \\
\text { perform? }\end{array}$ & $\begin{array}{l}5-15 \\
\min \end{array}$ & $\begin{array}{l}\text { Average } 7- \\
9 \mathrm{~min}\end{array}$ & $\leq 1 \mathrm{~min}$ & $30 \mathrm{~min}$ & $2-4 \min$ & Not stated & $\begin{array}{l}\text { Average } \\
7.8 \mathrm{~min}\end{array}$ & $\begin{array}{l}\text { Average } \\
114-202 \mathrm{~s}\end{array}$ & $\begin{array}{l}\text { Average } \\
30 \text { min }\end{array}$ & $\begin{array}{l}3-4 \\
\min \end{array}$ \\
\hline
\end{tabular}

ADOH: Activities of Daily Oral Hygiene, BOHSE: Brief Oral Health Status Examination, DHR: Dental Hygiene Registration, GOHAI: Geriatric Oral Health Assessment Index, MPS: Mucosal Plaque Score, OAS: Oral Assessment Sheet, OHAT: Oral Health Assessment Tool, OHSTNP: Oral Health Screening Tool for Nursing Personnel, RAI-MDS: Resident Assessment Instrument-Minimum Data Set, ROAG-J: Revised Oral Assessment Guide-Jonkoping

$[57,68]$. GOHAI is an internationally recognised tool, used in China, France, Sweden, United States, Netherlands, and Turkey [57, 59]. RAI-MDS has been implemented in the United States and Canada and can be used in different health care settings such as rehabilitative units, acute care, home care, and palliative care [62]. OHAT and BOHSE are widely accepted, validated, and user-friendly tools to be administered by non-dental personnel in American and Australian aged care facilities [24, 34]. Responsiveness of the oral health assessment instrument was measured in only one instrumentoral/dental items of RAI-MDS. There was no significant change in resident's oral/dental problems over 6 years from 2007 to 2012 [66].

\section{Reporting quality of identified instrument studies}

Among the 10 identified studies describing the oral health assessment instruments, the summary of reporting guidelines for survey research is compiled in Table 4. All studies adequately described the study objectives, results, and interpretation and discussion of the findings. Methodological concerns such as data entry, replication, and questionnaire administration were not described in all identified studies. Similarly, only one study discussed the role of response rate, non-response rate, and its calculation [53]. Furthermore, all studies provided information on the development of research tool except one [36], description of research tool except one [56], scoring methods except one [55], and reliability and validity except two $[35,56]$; whereas, five studies $[31,34,36,53$, $55]$ reported the instrument pre-testing features.

\section{Discussion}

The objectives of this systematic review were to provide a summary on the development and characteristics of oral health assessment instruments currently used by non-dental professionals for nursing home residents, and to perform a critical appraisal of the psychometric properties related to validity, reliability, feasibility, generalisability, and responsiveness of these instruments. We found ten oral health assessment instruments that were developed and tested to assess oral health of nursing home residents. Most of the instruments were developed by a panel of experts in geriatrics, dentistry, nursing, and in consultation with the users. However, narrow content, poorly defined constructs for measurement, and psychometric weaknesses were identified in the oral health assessment instruments.

A wide variation in measurement content was found across the oral health assessment instruments. OAS [54] and OHSTNP [31] measure oral function; DHR [52] and MPS [36] measure dental plaque; $\mathrm{ADOH}$ [35] measure oral self-care function; OHAT [43], BOHSE [34], and ROAG-J [56] measure oral health status in terms of lips, gums, tongue, saliva, tissues, natural/artificial teeth and cleanliness; RAI-MDS [55] measures oral/dental and nutritional status; and GOHAI [53] measures oral health conditions and psycho-social and functional problems. However, oral health encompasses the condition of individual's teeth and gums, and the health of the muscles and bones present in the mouth [2]. This indicates that none of the instruments were able to comprehensively measure all aspects of oral health.

Three major approaches for assessing oral health were identified: performance-based assessment, direct inspection of the oral health status, and interview measures. Performance-based assessment provides a quantitative measurement of an individual's ability to perform oral health related activities. However, a limitation of this approach is that it may fail to differentiate incapable or uninterested individuals [68]. As tools such as $\mathrm{ADOH}$ focus on self-care activities, a high score does not necessarily indicate a good oral health, especially in individuals with poor oral hygiene and inadequate oral health literacy [35]. Direct inspection integrates the inspection of lips, gums, teeth, tissues, tongue, and mucous membranes for any signs of oral problems. However, an unbiased observation relies largely on the human 
Table 4 Survey reporting quality of identified studies describing oral health assessment instruments

\begin{tabular}{|c|c|c|c|c|c|c|c|c|c|}
\hline Reporting items & $\begin{array}{l}\text { ADOH } \\
{[35]}\end{array}$ & $\begin{array}{l}\text { BOHSE } \\
{[34]}\end{array}$ & $\begin{array}{l}\text { DHR } \\
{[52]}\end{array}$ & $\begin{array}{l}\text { GOHAI } \\
{[53]}\end{array}$ & $\begin{array}{l}\text { MPS } \\
{[36]}\end{array}$ & $\begin{array}{l}\text { OAS } \\
{[54]}\end{array}$ & $\begin{array}{l}\text { OHAT } \\
\text { [43] }\end{array}$ & $\begin{array}{l}\text { OHSTNP } \\
\text { [31] }\end{array}$ & $\begin{array}{l}\text { RAI-MDS } \\
\text { [55] }\end{array}$ \\
\hline \multicolumn{10}{|l|}{ Background } \\
\hline Literature review & $P$ & A & A & A & $P$ & A & A & $P$ & A \\
\hline Explicit research question & I & A & A & A & । & । & A & A & A \\
\hline Clear study objectives & $A$ & A & $A$ & A & $A$ & A & $A$ & $A$ & A \\
\hline
\end{tabular}

\section{Methods}

Methods of data analysis
Questionnaire administration
Location of data collection
Dates of data collection
Methods sufficiently described for
replication
Methods of data entry
Sample selection

$$
\begin{aligned}
& \text { Sample size calculation } \\
& \text { Method of sample selection } \\
& \text { Description of population and } \\
& \text { sample frame }
\end{aligned}
$$

\section{Research Tool}

Description of research tool
Development of research tool
Instrument pretesting
Instrument reliability and validity
Scoring methods

\section{Results}

Results of research presented

Results address objectives

Generalisability

\begin{tabular}{|c|c|c|c|c|c|c|c|c|c|c|}
\hline Interpret and discuss findings & A & A & $A$ & A & A & A & A & A & A & A \\
\hline Conclusions and recommendations & I & $P$ & $P$ & $P$ & । & $P$ & $P$ & $P$ & 1 & $P$ \\
\hline Limitations & I & $P$ & A & । & । & । & । & I & I & । \\
\hline \multicolumn{11}{|l|}{ thics and discourse } \\
\hline Consent & I & A & A & । & । & A & A & A & I & A \\
\hline Sponsorship & । & A & $A$ & A & । & । & A & A & A & A \\
\hline Research ethics approval & 1 & 1 & 1 & 1 & । & A & A & A & 1 & A \\
\hline
\end{tabular}

\section{Response rate}

Response rate stated
Response rate calculated
Discussion of nonresponse
Missing data

\section{Interpretation and discussion}

A: Adequate, P: Partial, I: Inadequate. ADOH: Activities of Daily Oral Hygiene, BOHSE: Brief Oral Health Status Examination, DHR: Dental Hygiene Registration, GOHAl: Geriatric Oral Health Assessment Index, MPS: Mucosal Plaque Score, OAS: Oral Assessment Sheet, OHAT: Oral Health Assessment Tool, OHSTNP: Oral Health Screening Tool for Nursing Personnel, RAI-MDS: Resident Assessment Instrument-Minimum Data Set, ROAG-J: Revised Oral Assessment Guide-Jonkoping 
judgement and assessment accuracy may be affected by the variability in examiner [69]. Interview measures is a cost-effective way to collect information on oral health problems of cognitively intact residents; however, it has limited applications in RACFs. Moreover, the chances of oral symptoms being omitted, under-rated, and exaggerated by the examiner in both direct inspection and interview approaches may create discrepancy leading to false conclusions [70].

The variation in cognitive level and behaviour problems among nursing home residents evolve complexity in oral health screening [71]. Moreover, challenges may arise due to the lack of communication, co-operation, and self-reporting [26]. Oral care can be improved in moderately impaired residents by targeting nurses and carers to enhance their oral health skills; whereas, stageappropriate palliative oral care should be considered for severely impaired residents [72]. For unimpaired individuals with the ability to learn self-care skills, oral care function can be regained and maintained. Therefore, there is a need of an easy instrument to evaluate oral self-care of residents with cognitive impairment and such context should be considered through scientific approaches and research [73]. Instruments such as BOHSE [34], OHAT [43], and MPS [36] can be used for both cognitively impaired and unimpaired nursing home residents; GOHAI [53] is only applicable for cognitively unimpaired residents.

Methodological issues need to be reported transparently in the research process as it helps to assess the strengths and weaknesses and allow refinement of the instrument [50]. The reporting quality of the studies included in this review demonstrate mixed findings. Some domains such as background, results and its interpretation, and discussion were reported thoroughly; however, domains related to the methodological features were addressed inadequately. In most studies, the response rate and scoring issues of the instruments were not mentioned. Overall, all studies demonstrated inadequate compliance in reporting guidelines, which indicates possible advancement in developing oral health assessment instruments via further research.

Out of the ten identified instruments, only eight provided quality assessment on the basis of validity, reliability, feasibility and generalisability. However, the reporting of psychometric properties lacked explicit explanation on the concept of development of many instruments, which limits their methodological quality. Three out of the ten identified instruments- BOHSE [34], DHR [52], and OHAT [43] reported good methodological quality on at least one assessment criteria and appeared to be valid and reliable assessment tools for non-dental professionals to assess the oral health of nursing home residents. In a systematic review conducted in 2005, the authors found BOHSE as the most validated and reliable oral health assessment tool [24]. Although OHAT was developed as a modified form of BOHSE [43], oral health measurement categories of OHAT such as saliva, oral cleanliness, and dental pain require more investigation [43]. Similarly, OHSTNP [31], OAS [54], ADOH [35], RAI-MDS [55], and MPS [36] executed poor methodological quality of oral health measurement as the studies lacked comprehensive reflection of items to be measured (i.e. oral health). Furthermore, nurses were less proficient in accurately assessing lips, saliva, gums, mucosal membrane status, and chewing difficulty due to the lack of fundamental dental knowledge. Three studies [58, 60, 74] reported on the validation of GOHAI and three studies $[53,57,61]$ described its reliability and validity. Nevertheless, further research is necessary to review some of the items and to determine the sensitivity of the instruments to changes due to interventions over time.

Non-dental professionals can perform oral health assessment and provide referral services for nursing home residents, when supported with adequate resources and training [75]. Although nursing staff and carers of RACFs recognise the importance of oral health assessment, they have raised concerns on time commitment [31]. Moreover, inadequate knowledge among nondental professionals increases the risk of underestimation and overestimation of the symptoms leading to inappropriate scoring [70]. Therefore, some basic training for non-dental professionals on the specific signs and symptoms indicating oral infections and diseases is required [76]. Apart from training, enhancement of oral health knowledge of non-dental professionals is necessary. Provision of educational programs, use of diverse teaching formats, and regular reinforcement by a dental hygienist are found to be effective measures in enhancing the oral health of residents [77]. Moreover, some items of the oral health assessment instruments may require revision so that non-dental professionals can understand and administrate it easily, and further consideration is necessary in relation to frail and older adults in RACFs [78].

This systematic review has several strengths worth reporting. First, it provides valuable insight into the development, characteristics, and psychometric properties of oral health assessment instruments currently used by non-dental professionals for nursing home residents. Second, this review was conducted and reported in compliance with guidelines such as PRISMA and SURGE. Third, a structured methodological framework was used to retrieve data and critically appraise the existing oral assessment tools. Finally, the findings of our review may provide essential information for both dental and nondental professionals, which may aid in the successful 
collaboration of both professionals to ensure better oral health outcomes for the geriatric population residing in RACFs.

There are a few limitations of this review. First, articles published in languages other than English were not included and it is therefore possible that we may have missed some instruments published in a non-English language. Second, we may have missed some relevant publications despite following the PRISMA guidelines and using a combination of specific $\mathrm{MeSH}$, terms and keywords related to oral health, the geriatric population, and RACFs. Finally, two studies were removed due to accessibility issues despite repeated attempts to contact the authors, which could have provided valuable information. Nonetheless, this review presents an essential finding that none of the identified instruments were able to comprehensively measure all aspects of oral health. Further research is required on the development of valid and reliable instruments particularly for non-dental professionals that addresses the complexity, psychometric gaps, and appropriate content suitable for individuals utilising the tool.

\section{Conclusions}

Older adults residing in nursing homes are at a particularly high risk of developing oral health problems. Research highlights the importance of non-dental professionals such as nursing and care staff in oral health assessment and promotion in residential settings. This systematic review succeeded in providing a summary on the development and characteristics of oral health assessment instruments currently used by nondental professionals for nursing home residents and performing a critical appraisal of the psychometric properties of these instruments. The measurement content varied widely across the ten identified instruments, and none were able to comprehensively measure all aspects of oral health. Three measurement approaches were identified: performance- based assessment, direct inspection of the oral health status, and interview measures. Only eight instruments provided quality assessment on the basis of validity, reliability, feasibility and generalisability. However, three instruments- BOHSE, DHR, and OHAT appeared to be valid and reliable assessment tools for use by non-dental professionals to assess the oral health of nursing home residents. Nonetheless, continuous development of instruments is essential to address the psychometric gaps and embrace the complete spectrum of oral health. Moreover, there is a need of a valid, reliable, and more comprehensive tool to assess the oral health, identify the oral health needs, and generate positive outcomes in maintaining long-term oral health of the geriatric population residing in nursing homes.

\section{Supplementary Information}

The online version contains supplementary material available at https://doi. org/10.1186/s12877-020-01989-8.

Additional file 1: Appendix 1. Prisma Checklist

Additional file 2: Appendix 2. PICOS (Search terms)

Additional file 3: Appendix 3. MEDLINE search strategy

Additional file 4: Appendix 4. Reasons for exclusion of studies

\section{Abbreviations}

ADOH: Activities of Daily Oral Hygiene; BOHSE: Brief Oral Health Status Examination; DHR: Dental Hygiene Registration; DMFT: Decayed-MissingFilled-Teeth; GOHAl: Geriatric Oral Health Assessment Index; MPS: Mucosal Plaque Score; OAG: Oral Assessment Guide; OAS: Oral Assessment Sheet; OHAT: Oral Health Assessment Tool; OHI-S: Simplified Oral Hygiene Index OHSTNP: Oral Health Screening Tool for Nursing Personnel; PRIS MA: Preferred Reporting Items for Systematic Reviews and Meta-Analysis; PICOS: Population Intervention/Exposure Comparator Outcome Study Design; RAI-MDS: Resident Assessment Instrument-Minimum Data Set; ROAG: Revised Oral Assessment Guide; ROAG-J: Revised Oral Assessment Guide-Jonkoping; RACFs: Residential Aged Care Facilities; SLUMS: Saint Louis University Mental Status Examination; SURGE: Reporting Guidelines for Survey Research; THROAT: The Holistic and Reliable Oral Assessment Tool

\section{Acknowledgements}

We acknowledge the assistance of Ms. Katrina Chaudhary, School of Health Sciences librarian at Western Sydney University, in developing and testing the search strategy. We would like to acknowledge Mr. Muhammad El-Hajje, Dr. Narendar Manohar, and Associate Professor Ajesh George who were involved in the initial stages of conceptualising the research questions.

\section{Authors' contributions}

RT and AA conceptualised the research questions for the review. RT developed the search strategy and drafted the initial manuscript. AA and RC critically revised the initial and final versions of the manuscript. All authors approved the final manuscript as submitted.

\section{Funding}

AA was supported by Australian National Health and Medical Research Council Grants 1033213, 1069861, and 113475. The views expressed are those of the authors and not necessarily those of the Department of Health. The NHMRC had no role in the design and conduct of the study; collection, management, analysis, and interpretation of the data; preparation, review, or approval of the manuscript; and decision to submit the manuscript for publication.

\section{Availability of data and materials}

The datasets generated during and/or analysed during the current study are available from the corresponding author on reasonable request.

Ethics approval and consent to participate

Not applicable.

\section{Consent for publication}

Not applicable.

\section{Competing interests}

The authors declare that they have no competing interests.

\section{Author details}

${ }^{1}$ School of Health Sciences, Western Sydney University, Campbelltown Campus, Locked Bag 1797, Penrith, NSW 2751, Australia. ${ }^{2}$ School of Medicine, Western Sydney University, Campbelltown Campus, Locked Bag 1797, Penrith, NSW 2751, Australia. ${ }^{3}$ Translational Health Research Institute, Western Sydney University, Locked Bag 1797, Penrith, NSW 2751, Australia. ${ }^{4}$ Oral Health Services, Sydney Local Health District and Sydney Dental Hospital, NSW Health, Surry Hills, NSW 2010, Australia. ${ }^{5}$ Discipline of Child and Adolescent Health, Sydney Medical School, Faculty of Medicine and Health, The University of Sydney, Westmead, NSW 2145, Australia. 
Received: 10 February 2020 Accepted: 25 December 2020 Published online: 09 January 2021

\section{References}

1. Tran TD, Krausch-Hofmann S, Duyck J, de Almeida MJ, De Lepeleire J, Declerck D, et al. Association between oral health and general health indicators in older adults. Sci Rep. 2018:8:8871.

2. Australian Institute of Health and Welfare. Oral health and dental care in Australia. Canberra: Australian Institute of Health and Welfare; 2020.

3. Reigle JA, Holm K. Knowledge of oral health of nursing staff caring for disadvantaged older people. J Nurs Educ Pract. 2016;6:31-8.

4. Gil-Montoya JA, de Mello ALF, Barrios R, Gonzalez-Moles MA, Bravo M. Oral health in the elderly patient and its impact on general well-being: a nonsystematic review. Clin Interv Aging. 2015;10:461-7. https://doi.org/10. 2147/CIA.S54630

5. Nascimento GG, Leite FR, Vestergaard P, Scheutz F, Lopez R. Does diabetes increase the risk of periodontitis? A systematic review and meta-regression analysis of longitudinal prospective studies. Acta Diabetol. 2018;55:653-67.

6. Salamone K, Yacoub E, Mahoney A-M, Edward K-L. Oral care of hospitalised older patients in the acute medical setting. Nurs Res Pract. 2013;2013.

7. Ghezzi EM, Kobayashi K, Park DY, Srisilapanan P. Oral healthcare systems for an ageing population: concepts and challenges. Int Dent J. 2017:67:26-33.

8. Janssens B, Petrovic M, Jacquet W, Schols JM, Vanobbergen J, De Visschere L. Medication use and its potential impact on the oral health status of nursing home residents in Flanders (Belgium). J Am Med Dir Assoc 2017;18: 809. e1-. e8.

9. Petersen PE, Kandelman D, Arpin S, Ogawa H. Global oral health of older people-call for public health action. Community Dent Health. 2010;27:257-67.

10. Chrisopoulos S, Harford J, Ellershaw A. Oral health and dental care in Australia: key facts and figures 2015: Australian Institute of Health and Welfare; 2016

11. Griffin SO, Jones JA, Brunson D, Griffin PM, Bailey WD. Burden of oral disease among older adults and implications for public health priorities. Am J Public Health. 2012;102:411-8.

12. Ramsay S, Whincup P, Watt R, Tsakos G, Papacosta A, Lennon L, et al. Burden of poor oral health in older age: findings from a population-based study of older British men. BMJ Open. 2015:5:e009476.

13. Australian Institute of Health and Welfare. The National Survey of Adult Oral Health 2004-06. 2008.

14. Petersen $\mathrm{PE}$, Ogawa $\mathrm{H}$. Promoting Oral health and quality of life of older people-the need for public health action. Oral Health Prev Dent. 2018;16: 113-24

15. Coker E, Ploeg J, Kaasalainen S. The effect of programs to improve oral hygiene outcomes for older residents in long-term care: a systematic review. J Gerontol Nurs. 2014;7:87-100.

16. Weening-Verbree L, Huisman-de Waal G, van Dusseldorp L, van Achterberg T, Schoonhoven L. Oral health care in older people in long term care facilities: a systematic review of implementation strategies. Int J Nurs Stud. 2013;50:569-82.

17. Monaghan N, Karki A, Playle R, Johnson I, Morgan M. Measuring oral health impact among care home residents in Wales. Community Dent Health. 2017;34:14-8. https://doi.org/10.1922/CDH_3950Morgan05.

18. Hearn L, Slack-Smith L. Oral health care in residential aged care services: barriers to engaging health-care providers. Aust J Prim Health. 2015;21:148-56.

19. Slack-Smith L, Durey A, Scrine C. Successful ageing and oral health: incorporating dental professionals into aged care facilities. 2016.

20. Gulati NK. Overcoming Oral hygiene challenges in the nursing home. Caring for the Ages. 2017;18:3.

21. Willumsen, Karlsen L, Næss R, Bjørntvedt S. Are the barriers to good oral hygiene in nursing homes within the nurses or the patients? Gerodontology. 2012;29:e748-e55.

22. Andersson, Renvert S, Sjogren P, Zimmerman M. Dental status in nursing home residents with domiciliary dental care in Sweden. Community Dent Health. 2017;34:203-7.

23. Petersen PE, Yamamoto T. Improving the oral health of older people: the approach of the WHO global Oral health Programme. Community Dent Oral Epidemiol. 2005;33:81-92.

24. Chalmers, Pearson A. A systematic review of oral health assessment by nurses and carers for residents with dementia in residential care facilities. Spec Care Dentist. 2005;25:227-33.
25. Eldh AC, Olai L, Jönsson B, Wallin L, Denti L, Elf M. Supporting first-line managers in implementing oral care guidelines in nursing homes. Nord J Nurs Res. 2018;38:87-95. https://doi.org/10.1177/2057158517713379.

26. Foley N, Affoo R, Siqueira W, Martin R. A systematic review examining the oral health status of persons with dementia. J Am Geriatr Soc. 2015;63:S36

27. Dolhaniuk I, Moser-Siegmeth V, Dorner C, Hammerle S, Terzic A, Tarnawski $U$, et al. Promoting oral health and oral hygiene in a geriatric nursing home-a pilot. Eur Geriatr Med. 2017;8:S209-S10.

28. Siegel E, Cations M, Wright C, Naganathan V, Deutsch A, Aerts L, et al. Interventions to improve the oral health of people with dementia or cognitive impairment: a review of the literature. J Nutr Health Aging. 2017; 21:874-86.

29. Shay K. Who is responsible for a nursing home resident's daily oral care? J Am Geriatr Soc. 2007:55:1470-1. https://doi.org/10.1111/j.1532-5415.2007. 01280.x.

30. Chalmers, Spencer AJ, Carter KD, King PL, Wright C. Caring for oral health in Australian residential care. Dental statistics and research series. 2009:48.

31. Tsukada S, Ito K, Stegaroiu R, Shibata S, Ohuchi A. An oral health and function screening tool for nursing personnel of long-term care facilities to identify the need for dentist referral without preliminary training. Gerodontology. 2017:34:232-9. https://doi.org/10.1111/ger.12255.

32. Eilers J, Berger AM, Petersen M, editors. Development, testing, and application of the oral assessment guide. Oncol Nurs Forum; 1988.

33. Dickinson $H$, Watkins $C$, Leathley $M$. The development of the THROAT: the holistic and reliable oral assessment tool. Clin Eff Nurs. 2001;5:104-10. https://doi.org/10.1054/cein.2001.0221.

34. Kayser-Jones J, Bird WF, Paul SM, Long L, Schell ES. An instrument to assess the oral health status of nursing home residents. Gerontologist. 1995;35: 814-24.

35. Bauer JG. The index of ADOH: concept of measuring oral self-care functioning in the elderly. Spec Care Dentist. 2001;21:63-7.

36. Henriksen BM, Ambjørnsen E, Axéll TE. Evaluation of a mucosal-plaque index (MPS) designed to assess oral care in groups of elderly. Spec Care Dentist. 1999:19:154-7.

37. Pearson A, Chalmers J. Oral hygiene care for adults with dementia in residential aged care facilities. Int J Evid Base Healthc. 2004;2:65-113.

38. Thorndike RM. Measurement and evaluation in psychology and education. 7th ed. ed. Upper Saddle River, N.J.: Upper Saddle River, N.J. : Pearson Merrill Prentice Hall; 2005.

39. Merom D, John JR. Measurement issues in quantitative research. Handbook of Research Methods in Health Social Sciences. 2018:1-18.

40. Portney LG. Foundations of clinical research : applications to practice. 2nd ed. ed. Watkins MP, editor. Upper Saddle River, NJ: Upper Saddle River, NJ : Prentice Hall; 2000.

41. Cardol M, Beelen A, van den Bos GA, de Jong BA, de Groot IJ, de Haan RJ. Responsiveness of the impact on participation and autonomy questionnaire. Arch Phys Med Rehabil. 2002;83:1524-9.

42. Andersson, Hallberg IR, Renvert S. Inter-rater reliability of an oral assessment guide for elderly patients residing in a rehabilitation ward. Spec Care Dentist. 2002;22:181-6.

43. Chalmers, King PL, Spencer AJ, Wright FAC, Carter KD. The oral health assessment tool--validity and reliability. Aust Dent J. 2005;50:191-9.

44. Moher D, Liberati A, Tetzlaff J, Altman DG. Preferred reporting items for systematic reviews and meta-analyses: the PRISMA statement. Ann Intern Med. 2009:151:264-9.

45. Booth A, Clarke M, Dooley G, Ghersi D, Moher D, Petticrew M, et al. The nuts and bolts of PROSPERO: an international prospective register of systematic reviews. Systematic reviews. 2012;1:2-. doi: https://doi.org/10 1186/2046-4053-1-2

46. Costantino G, Montano N, Casazza G. When should we change our clinical practice based on the results of a clinical study? Searching for evidence: PICOS and PubMed. Intern Emerg Med. 2015;10:525-7.

47. Jolles BM, Buchbinder R, Beaton DE. A study compared nine patient-specific indices for musculoskeletal disorders. J Clin Epidemiol. 2005:58:791-801.

48. Jordan JE, Osborne RH, Buchbinder R. Critical appraisal of health literacy indices revealed variable underlying constructs, narrow content and psychometric weaknesses. J Clin Epidemiol. 2011;64:366-79. https://doi.org/ 10.1016/j.jclinepi.2010.04.005.

49. Bennett C, Khangura S, Brehaut JC, Graham ID, Moher D, Potter BK, et al. Reporting guidelines for survey research: an analysis of published guidance and reporting practices. PLoS Med. 2011;8:e1001069. 
50. Altin SV, Finke I, Kautz-Freimuth S, Stock S. The evolution of health literacy assessment tools: a systematic review. BMC Public Health. 2014;14:1207.

51. Bowen DJ, Kreuter M, Spring B, Cofta-Woerpel L, Linnan L, Weiner D, et al. How we design feasibility studies. Am J Prev Med. 2009;36:452-7.

52. Fjeld KG, Eide $H$, Mowe M, Hove LH, Willumsen T. Dental hygiene registration: development, and reliability and validity testing of an assessment scale designed for nurses in institutions. J Clin Nurs. 2017;26: 1845-53. https://doi.org/10.1111/jocn.13452.

53. Atchison KA, Dolan TA. Development of the geriatric oral health assessment index. J Dent Educ. 1990:54:680-7.

54. Shizuko Y, Masanori N, Takaharu G, Masami Y, Yasuhiko S. Development of an Oral assessment sheet for evaluating older adults in nursing homes. Res Gerontol Nurs. 2017;10:234-9. https://doi.org/10.3928/19404921-20170621-04

55. Morris J. Designing the National Resident Assessment Instrument for nursing homes. Gerontologist. 1990;30:293-307.

56. Johansson I, Jansson H, Lindmark U. Oral health status of older adults in Sweden receiving elder care: findings from nursing assessments. Nurs Res. 2016;65:215-23. https://doi.org/10.1097/NNR.0000000000000158.

57. Ergül S, Akar GC. Reliability and validity of the geriatric Oral health assessment index in Turkey. J Gerontol Nurs. 2008;34:33-9. https://doi.org/ 10.3928/00989134-20080901-05.

58. Wong MC, Liu JK, Lo EC. Translation and validation of the Chinese version of GOHAl. J Public Health Dent. 2002;62:78-83.

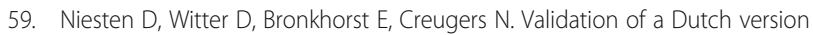
of the Geriatric Oral Health Assessment Index (GOHAI-NL) in caredependent and care-independent older people. BMC geriatrics. 2016;16:53-. doi: https://doi.org/10.1186/s12877-016-0227-0.

60. Tubert-Jeannin S, Riordan P, Morel-Papernot A, Porcheray S, Saby-Collet S. Validation of an oral health quality of life index (GOHAl) in France. Community Dent Oral Epidemiol. 2003;31:275-84.

61. Hägglin C, Berggren U, Lundgren J. A Swedish version of the GOHAl index. Psychometric properties and validation. Swed Dent J. 2005;29:113-24.

62. Hutchinson AM, Milke DL, Maisey S, Johnson C, Squires JE, Teare G, et al. The resident assessment instrument-minimum data set 2.0 quality indicators: a systematic review. BMC Health Serv Res. 2010;10:166.

63. Thai PH, Shuman SK, Davidson GB. Nurses' dental assessments and subsequent care in Minnesota nursing homes. Spec Care Dentist. 1997;17:13-8.

64. Poss J, Jutan N, Hirdes J, Fries B, Morris J, Teare G, et al., editors. A review of evidence on the reliability and validity of Minimum Data Set data. Healthcare Management Forum; 2008: SAGE publications Sage CA: Los Angeles, CA.

65. Krausch-Hofmann S, De Almeida MJ, Declerck D, Declercq A, De Lepeleire J, Tran TD, et al. The oral health-related section of the interRAl: evaluation of test content validity by expert rating and assessment of potential reasons for inaccurate assessments based on focus group discussions with caregivers. Gerodontology. 2019;36:382-94. https://doi.org/10.1111/ger.12421.

66. Hoben M, Poss JW, Norton PG, Estabrooks CA. Oral/dental items in the resident assessment instrument - minimum data set 2.0 lack validity: results of a retrospective, longitudinal validation study. Popul Health Metrics. 2016; 14:1-13. https://doi.org/10.1186/s12963-016-0108-y.

67. Landis JR, Koch GG. The measurement of observer agreement for categorical data. Biometrics. 1977:159-74.

68. Chen X, Zimmerman S, Potter GG, Sloane PD, Cohen LW, Reed D. Assessment of dentally related function in individuals with cognitive impairment: the dental activities test. J Am Geriatr Soc. 2017;65:580-5.

69. Quinn B, Stone R, Uhlenhopp M, McCann S, Blijlevens N. Ensuring accurate oral mucositis assessment in the European group for blood and marrow transplantation prospective oral mucositis audit. Eur J Oncol Nurs. 2007;11: S10-S8.

70. Hinds PS, Schum L, Srivastava DK. Is clinical relevance sometimes lost in summative scores? West J Nurs Res. 2002;24:345-53.

71. Rantzow V, Andersson P, Lindmark U. Occurrence of oral health problems and planned measures in dependent older people in nursing care. J Clin Nurs. 2018;27:4381-9. https://doi.org/10.1111/jocn.14584.

72. Chen, Kistler CE. Oral health care for older adults with serious illness: when and how? J Am Geriatr Soc. 2015;63:375-8.

73. Jablonski RA. Examining oral health in nursing home residents and overcoming mouth care--resistive behaviors. Ann Longterm Care. 2010;18:21-6.

74. Othman WNW, Muttalib KA, Bakri R, Doss JG, Jaafar N, Salleh NC, et al. Validation of the geriatric oral health assessment index (GOHAl) in the Malay language. J Public Health Dent. 2006;66:199-204.
75. Poudel P, Griffiths R, Wong WW, Arora A, George A. Knowledge and practices of diabetes care providers in oral health care and their potential role in oral health promotion: a scoping review. Diabetes Res Clin Pract. 2017;130:266-77.

76. Jordan R, Sirsch E, Gesch D, Zimmer S, Bartholomeyczik S. Improvement of oral health care in geriatric care by training of nurses and nursing assistants for the elderly. Pflege. 2012;25:97-105.

77. Miegel K, Wachtel T. Improving the oral health of older people in long-term residential care: a review of the literature. Int J Older People Nursing. 2009;4 97-113. https://doi.org/10.1111/j.1748-3743.2008.00150.x.

78. Barnett T, Hoang H, Stuart J, Crocombe L, Bell E. Utilisation of oral health services provided by non-dental health practitioners in developed countries: a review of the literature. Community Dent Health. 2014;31:224-33.

\section{Publisher's Note}

Springer Nature remains neutral with regard to jurisdictional claims in published maps and institutional affiliations.
Ready to submit your research? Choose BMC and benefit from:

- fast, convenient online submission

- thorough peer review by experienced researchers in your field

- rapid publication on acceptance

- support for research data, including large and complex data types

- gold Open Access which fosters wider collaboration and increased citations

- maximum visibility for your research: over $100 \mathrm{M}$ website views per year

At BMC, research is always in progress.

Learn more biomedcentral.com/submissions 